\title{
Déconfiner l'expertise sur les faibles doses de rayonnements ionisants
}

\section{The opening of the expertise on the small doses of ionizing radiations}

\author{
Elisabeth Remy ${ }^{(1)}$, Jacqueline Estades ${ }^{(2)}$ \\ (1) INRA/INRA/SAD-APT, 16 rue Claude Bernard, 75231 Paris Cedex 05, France \\ elisabeth.remy@inapg.inra.fr \\ (2) UMR GAEL Grenoble, INRA/Université Pierre-Mendes-France, BP 47, 38040 Grenoble Cedex, France \\ estades@inra.fr
}

Résumé - Les riverains de sites nucléaires oscillent entre des positions très contrastées au sujet de l'impact des faibles doses de rayonnements ionisants. L'expertise conduite par le Groupe radioécologie Nord-Cotentin nous permet d'interroger la coupure établie entre spécialistes et «profanes » dans l'évaluation des risques. Apparaissent ainsi les conditions nécessaires pour bâtir une expertise pluraliste et crédible.

Mots clés - Experts, «profanes », sociologie, faibles doses, risques collectifs

Abstract - People who lived near nuclear installations have very contrasted points of view about the impact of low doses of ionizing radiations. Through the expertise implemented by the "Groupe radioécologie Nord-Cotentin", we examine the usual opposition done between the specialists and the "laymen" about the assessment of risks. From this situation, we analyse the necessary conditions for constructing a pluralist and credible expertise.

Key words - Experts, laymen, Sociology, low doses of ionizing radiations, collective risks

\section{INTRODUCTION}

Les riverains de sites nucléaires oscillent entre des positions très contrastées au sujet de l'impact des faibles doses de rayonnements ionisants: s'en remettre aux experts du domaine, rejoindre les associations militantes et critiques ou adopter des stratégies d'évitement ${ }^{(1)}$. L'hypothèse avan-

(1) Cette recherche a été financée par le programme Risques collectifs et situations de crise cée dans une étude épidémiologique ${ }^{(2)}$ d'une relation causale entre le développement de leucémies et certaines habitudes de vie associées aux re-

du CNRS dirigé par C. Gilbert; se reporter à Estades J. \& Rémy E. (2003).

(2) Pobel D., Viel J.F. (1997) «Case-control study of leukaemia among young people near La Hague nuclear reprocessing plant : the environmental hypothesis revisited ", BMJ, Vol. 314, (101), January, p. 101-106. Voir aussi Viel J.F. (1998). 
jets des installations nucléaires est-elle fondée compte tenu de ce que l'on sait sur les effets des rayonnements? C'est pour apporter des éléments de réponse à cette question qu'un comité d'experts - le groupe radioécologie Nord-Cotentin (GRNC) - a initié un travail collectif réunissant des acteurs institutionnels et associatifs ainsi que les exploitants du secteur. La sélection des acteurs qui ont légitimité à participer à l'expertise est révélatrice de la mise en tension du statut et du rôle de l'expert aujourd'hui. Pour certains, l'expert scientifique serait devenu en quelque sorte l'homme à abattre puisqu'il dispose seul du droit d'assurer l'interface entre science et politique, excluant ainsi le non spécialiste de la scène experte. Une autre tendance vise à revaloriser les profanes comme s'il s'agissait de racheter leurs compétences trop longtemps sous estimées. Au-delà de ces oppositions, c'est la question des conditions de l'organisation d'une expertise pluraliste sur les risques qui est posée, dans un contexte global de rénovation de l'expertise des risques sanitaires et environnementaux « qui doit rendre accessible aux responsables les connaissances scientifiques les plus actuelles sur l'origine ou l'ampleur des problèmes de santé et les moyens disponibles pour y répondre » (Dab, 1997 p. 199). En contrepoint, «les citoyens ont besoin d'interlocuteurs bien identifiés auxquels ils peuvent s'adresser en cas de menaces ressenties. II doit s'agir de professionnels détenteurs d'une responsabilité universelle de santé publique, valable quelle que soit la source de la menace. " (Dab, 1998 - p. 157). Dans cette perspective, bien que permettant l'accès des profanes à des données objectives, le spécialiste demeure l'épicentre et le garant d'un bon équilibre entre évaluation et gestion des risques pour un bénéfice social profitant à tous.

Mais, pour imposer le spécialiste comme la figure légitime exclusive de l'expertise, il est nécessaire de fabriquer la figure du profane dotée des attributs contraires. Sans disqualifier l'apport des scientifiques dans l'évaluation des risques, nous interrogeons la coupure établie entre spécialistes et profanes au nom du primat de la rationalité savante. Le cas de l'expertise liée aux faibles doses de rayonnements ionisants montre que toutes les ressources cognitives mobilisables pour objectiver des facteurs de risques relèvent autant de savoirs généraux que de savoirs locaux. Renvoyer spécialistes et profanes à des régimes d'action et à des mondes antinomiques, revient à affirmer que le savoir des spécialistes «qui fait aujourd'hui autorité n'est déterminé que par les phénomènes naturels, à partir de lois objectives, sans être le moins du monde imprégné par des hypothèses et des engagements de nature sociale. C'est ainsi que se trouve obscurcie la présence d'indéterminations et de contingences au cœur de la connaissance scientifique utilisée dans l'évaluation des risques d'environnement. Indéterminations et contingences endogènes s'en trouvent travesties en incertitudes exogènes de type déterministe, a priori plus accessible à un contrôle par la science » (Wynne, 1997 - p. 149-50). L'asymétrie entre spécialistes et profanes fondée sur une opposition entre objectivité du discours savant et subjectivité de l'opinion profane implique donc une disqualification 
ex ante de la contribution profane à une expertise des risques et confère au savoir scientifique le double statut de boîte noire et de boîte à outils quasi exclusive de l'expertise. Notre étude du GRNC souligne au contraire que dans l'activité d'expertise, le savoir local, l'énoncé scientifique et la prescription normative se mêlent, selon différentes modalités, pour constituer la substance même de l'évaluation et de la gestion des risques (Cambrosio et al., 1992).

Après avoir décrit comment le GRNC s'est organisé en collectif de travail (Section 2), nous analyserons les négociations et les compromis qu'implique une expertise ouverte (Section 3). Puis, à partir de l'exemple d'une campagne de mesures conduite dans la zone de la Hague, nous montrerons comment s'est construite une expertise pluraliste pour « apprivoiser » les faibles doses de rayonnements ionisants (Section 4).

\section{CONSTRUIRE LE COLLECTIF D'EXPERTS}

L'usine de retraitement de déchets nucléaires de La Hague est l'objet répété de polémiques (Chateauraynaud et Torny, 1999) qui jettent un doute sur la fiabilité des propos tenus par les spécialistes du domaine. L'étude épidémiologique de J.-F. Viel, largement relayée par la presse, suscite immédiatement de vives réactions. En juillet 1997, les ministres de la Santé et de l'Environnement français demandent à Annie Sugier de piloter un groupe d'experts $^{(3)}$ pour estimer, à partir d'une re-

(3) Ce comité fait suite à une autre commission d'experts qui a travaillé six mois sur cette question dans un climat interne très conflictuel. constitution des expositions provenant de différentes sources de rayonnements ionisants, le risque de leucémie attribuable à ces sources au cours de la période 1978-1996 pour les jeunes de 0 à 24 ans du Canton de BeaumontHague. Les missions de ce comité le GRNC - sont de deux ordres(4) : « apporter des éléments complémentaires d'information aux études épidémiologiques réalisées ou en cours dans le Nord-Cotentin »; et " éclairer les décisions à prendre sur la révision des textes réglementaires régissant le fonctionnement de l'usine de retraitement COGEMA-La Hague, en évaluant les expositions des groupes de population susceptibles d'être les plus exposés ".

La fixation de la composition des comités constitue une première étape importante pour le cadrage de l'expertise comme dispositif collectif dans la mesure où elle précise les acteurs, les métiers et les disciplines légitimes pour répondre à la commande.

\subsection{Négocier l'ouverture de l'expertise}

Dès son initiation, la présidente du GRNC engage des négociations avec ses tutelles pour ouvrir le comité à d'autres catégories d'acteurs que ceux habituellement entendus sur ces questions. "Je dis d'abord qu'on ne peut pas rester un groupe où il n'y a que

(4) Citées dans Groupe Radioécologie NordCotentin (1999) - Estimation des niveaux d'exposition aux rayonnements ionisants et des risques de leucémies associés de population du Nord-Cotentin, rapport de synthèse, Fontenayaux-Roses, Institut de Protection et de Sûreté Nucléaire. 
des exploitants et des institutionnels. Je pense qu'il faut ouvrir à la fois localement et au mouvement associatif (...). D'abord, ils (les opérateurs et les exploitants) me disent qu'ils sont contre ma proposition et, finalement, dès lors que les tutelles approuvent ma démarche, les exploitants s'inclinent (...). Donc, cela signifie que je peux constituer un groupe comprenant les experts qui sont parties prenantes et qui ont un avis sur l'affaire " (Entretien, IPSN, 1999) ${ }^{(5)}$.

Ainsi se trouveront associées à l'expertise des associations dont la contribution dépendra de leur histoire et de leur spécificité. Équipée d'un laboratoire d'analyses, I'ACRO(6) est une association orientée vers l'expertise et l'information du public en matière de surveillance de l'environnement à l'échelon régional. Ses membres veulent peser sur les processus de décision en associant expertise et actions militantes. Le GSIEN ${ }^{(7)}$ se montre très actif pour procéder à des exercices de vérification ${ }^{(8)}$. Ces associations veulent réinterroger les routines et les connaissances tacites détenues par les experts institutionnels et les opérateurs et qui finissent par

(5) Pour chaque extrait, nous précisons l'année de l'entretien et l'appartenance du locuteur conformément à la déontologie de la méthode d'enquête.

(6) ACRO : Association pour le Contrôle de la Radioactivité dans l'Ouest.

(7) GSIEN : Groupement de Scientifiques pour l'Information sur l'Energie Nucléaire.

(8) Cette association développe une information critique sur les orientations de l'industrie nucléaire et milite pour une expertise indépendante et la participation du public aux décisions importantes destinées à orienter ce domaine d'activités. prendre l'allure de certitudes: «On se dit... des gens qui font que ça tous les jours sont enfermés dans leur système, dans leur vision et il y a des choses qui échappent et parfois ce sont des gens qui ne sont pas forcément experts, qui sont simplement un peu observateurs, qui voient des choses énormes qui n'ont pas pu être vues ». L'enjeu de cette option organisationnelle est de favoriser les rapprochements entre des gens qui sont dans des métiers, des disciplines et des logiques différentes sans pour autant diluer l'objectif de l'expertise dans la recherche d'un consensus. Mais certains de ces acteurs sont habituellement dans des rapports conflictuels fortement teintés d'enjeux militants pour les uns, économiques pour les autres. II s'agit donc de leur montrer qu'ils ont un intérêt à coopérer pour cette « opération " au moment même où les pouvoirs publics réforment le contrôle et l'expertise du nucléaire.

\subsection{Miser sur le pluralisme}

La désignation de la composition d'un comité ne garantit en rien que ses membres parviendront à travailler ensemble s'ils ne se dotent pas de conditions favorables pour le faire. II leur faut donc s'organiser en collectif par rapport à deux fronts : un front intérieur pour accomplir leur tâche d'expert; et un front extérieur pour intégrer dans leur travail les actions et réactions de ce monde extérieur qui a les yeux braqués sur eux. Dès sa mise en place, le GRNC reconnaît un statut équivalent aux institutionnels et aux associatifs 
dans la conduite de l'étude exhaustive des données concernant les rejets, les mesures, les modèles et le calcul des doses et des risques liés aux installations nucléaires de façon à ouvrir les boîtes noires de la production des données à tous les membres du comité. "II ne s'agissait plus de se contenter de donner un résultat mais de dire comment on faisait et surtout de dire [aux opérateurs] « maintenant, vous donnez tous les chiffres que vous avez et c'est nous qui allons reprendre les calculs "; ça c'est une démarche qu'ils n'avaient jamais eue ".

La démarche d'ensemble retenue par le GRNC associe deux méthodologies complémentaires en validant les modèles par la comparaison de leurs résultats avec les mesures faites dans l'environnement : l'une consiste à mesurer les concentrations en radionucléides dans l'environnement et à estimer en conséquence l'impact sur des groupes de population compte tenu de leur localisation géographique et de leur utilisation de cet environnement; l'autre consiste, connaissant les rejets, à s'appuyer sur des modèles traduisant les mécanismes de dispersion et de reconcentration des radionucléides dans l'environnement jusqu'à l'homme.

Pour conduire un examen minutieux des éléments liés aux rejets radioactifs et suivre à la trace les données mobilisées dans les modèles et les calculs, les 50 membres du comité$^{(9)}$ se sont organisés en quatre groupes couvrant les rejets, les mesures, les modèles et les doses.

(9) Le groupe pleinier se réunit mensuellement. Chaque groupe thématique conduit son travail de manière autonome et un point est fait à la réunion mensuelle du groupe pleinier.
- « Le premier examine de façon critique les rejets déclarés par les exploitants des installations nucléaires du Nord-Cotentin et, si nécessaire, reconstitue les données manquantes ;

- le deuxième rassemble et interprète les mesures faites dans l'environnement par les différents intervenants (institutionnels et non institutionnels) depuis la mise en service des installations;

- le troisième compare entre eux les modèles et confronte les prévisions de ceux-ci avec les mesures faites dans l'environnement;

- Le quatrième identifie les groupes de population du Nord-Cotentin pertinents du point de vue épidémiologique ou dont les habitudes de vie les conduisent à être plus exposés aux sources d'origine nucléaire, afin d'évaluer le niveau moyen des expositions auxquelles ils ont été ou sont soumis; il évalue également le niveau moyen des expositions reçues du fait des autres sources de rayonnement (naturelles et médicales) et estime le risque correspondant à la somme de l'ensemble des expositions considérées »(10).

Même si les associatifs expriment à plusieurs reprises des difficultés d'accès aux discussions scientifiques et techniques, l'organisation du travail adoptée engage les exploitants à

(10) Extrait de la note méthodologique rédigée par la présidente du GRNC «Approche méthodologique du groupe radioécologie NordCotentin chargé de l'évaluation des doses et des risques dus aux expositions aux rayonnements de la population du Nord-Cotentin ». 
coopérer avec eux à travers une évaluation élargie du risque qui donne à ceux-ci la possibilité de s'immiscer dans la logique des calculs. Ainsi, une association très active dans la région comme l'ACRO est présente dans les quatre groupes de travail. Malgré une asymétrie initiale d'accès aux données scientifiques de la part des différents membres, l'organisation interne du GRNC a fait le pari de la possibilité d'un travail en commun entre les experts traditionnels du nucléaire et des intervenants extérieurs mobilisés localement ou nationalement sur ces questions dans des associations. Pour cela, le comité a développé une importante activité d'écriture à la fois collective et distribuée (compte-rendus détaillés des réunions, diffusion de toutes les observations sur chacun des rapports, rédaction des conclusions des travaux, etc.). La présidente prépare un premier texte afin de mettre en application sa ligne directrice qui vise à l'exhaustivité et à la compréhension de l'enregistrement de tous les points de vue. Ce premier projet est plus ou moins enrichi ou amendé par les animateurs secrétaires des groupes de travail. Puis la nouvelle version est revue ligne par ligne en réunion plénière pour introduire le plus de nuances possibles. C'est la base de la «négociation » entre les différents acteurs. À la suite de quoi, deux formes de diffusion écrites des désaccords sont intégrées dans les rapports de conclusion : I'internalisation de propositions alternatives dans le corps du texte et l'inscription des désaccords à la suite d'une conclusion commune. Ainsi, le GRNC a-t-il été capable de produire une expertise collective intégrant des avis critiques et des acteurs multiples.

\subsection{Dialoguer avec « les mères en colère »}

Comment le GRNC a-t-il procédé pour composer avec un extérieur parfois critique sans dévoyer le contenu de son travail car l'enjeu de son mandat est aussi de contribuer à apaiser ces turbulences? II a reconduit le schéma pluraliste de fonctionnement dans sa communication externe. Chacun des membres peut s'exprimer à l'extérieur sur le travail du groupe, y compris à des journalistes, à condition de ne pas anticiper sur la validation collective des résultats. Mais c'est le dialogue engagé entre le GRNC et une association locale, "les mères en colère », qui incarne le mieux cette dynamique entre un comité d'experts ouvert qui veut composer avec son environnement social et une population locale qui réagit à une situation problématique en s'organisant pour être partie prenante des débats. En février 1997, des mères de famille du Nord-Cotentin réagissent à la parution de l'étude de Jean-François Viel en créant un collectif afin de ne plus être écartelées entre des propos extrémistes et alarmistes ou hermétiques et rassurants. «Absurde! » avait titré un journal local au lendemain de la publication de l'étude de Viel. C'était l'analyse qu'en faisait aussi COGEMA(11). C'est vraiment ce mot-là qui sur le parking de l'école nous a vraiment motivées parce qu'on voulait à notre manière essayer de comprendre ce qui se passait et surtout avoir des informations claires, indépendantes, objectives. C'est parti d'un noyau de copines, de catégories professionnelles

(11) COGEMA : COmpagnie GEnérale des MAtières nucléaires 
très variées (professeurs, institutrices, infirmières, mères de famille, agricultrices) (Entretien, "Mères en colère ", 1999). Le collectif compte une quarantaine de femmes très actives dans tout le Nord-Cotentin et entre 300 à 350 sympathisants. Face aux associations très marquées, localement, comme les Verts et le CRILAN (Comité Régional d'Information de Lutte AntiNucléaire), l'idée que défend le collectif est de se démarquer de tous les lobbies anti ou pro nucléaire qu'elles jugent trop extrémistes. "Dans le groupe, il y a forcément des gens qui ont une certaine culture anti-nucléaire. Mais surtout, ce qu'on veut, c'est que «les mères en colère » soient bien démarquées de ces associations-là parce que c'est ouvert et parce que ça a permis à des femmes, qui jusqu'alors ne seraient pas allées au devant de l'information, de venir, de s'informer, de parler » (Idem).

Le GRNC a reconnu "les mères en colère » comme un interlocuteur extérieur important en le tenant informé de l'avancement de ses travaux. Réciproquement, l'association accorde du crédit au travail du GRNC et fait confiance à son organisation pour conduire une analyse fiable des facteurs de risque. Elle circule aussi parmi les familles et recueille des témoignages sur la perception des risques qu'elle transmet au GRNC pour élargir au maximum le champ d'expertise des risques sanitaires. "Quand on a fait signer les pétitions, combien de gens nous ont dit " moi j'ai de la famille, moi j'ai ci, moi j'ai ça, concernant la thyroïde" ". On sent vraiment qu'il y a quelque chose. Là, il y a le registre du cancer qui nous promet depuis des mois des études sur le cancer de la thyroïde et à chaque fois c'est repoussé, donc c'est pareil, on fait vraiment pression pour qu'ils nous sortent ces choses-là, pour améliorer le suivi sanitaire» (Idem). Les « mères en colère " constituent ainsi le vecteur central de la stratégie de construction de la crédibilité locale du GRNC, dans la mesure où elles sont à la fois un groupe de référence à informer et des informatrices dignes d'attention et d'écoute.

Le GRNC a donc joué la carte de l'ouverture aussi bien dans son organisation interne que dans ses relations avec l'extérieur. II a encouragé une communication et une information libérant l'expertise de la situation de blocage entre les positions de la contre-expertise militante et celles des opérateurs très vigilants quant à la sûreté de leurs installations. Pourtant des procédures réglementaires et des moyens importants sont mis en œuvre pour assurer le contrôle des installations et la surveillance de l'environnement. Les missions réglementaires des exploitants sont encadrées par des contrôles externes stricts. Néanmoins, les associations membres du GRNC considèrent qu'il n'y a pas équivalence entre la garantie de sécurité des installations et le suivi sanitaire de la population.

\section{PRODUIRE DES SAVOIRS EXPERTS}

Saisir les modalités d'interaction entre divers acteurs en situation d'expertise suppose d'entrer dans le cœur de l'évaluation des risques pour saisir ce que produit cette ouverture. Quels sont les savoirs qui auront droit de cité dans l'expertise? Comment 
s'immiscer dans la logique des calculs? À quelle évaluation du risque parvient-on?

\subsection{Prendre en compte un savoir local}

Un des membres associatifs du GRNC soumet au comité la question de la prise en compte d'une pratique locale comme l'épandage des algues dans le calcul des doses. "C'est une pratique qu'on a dans le NordCotentin, ... l'épandage d'algues sur les terrains qu'on utilise comme engrais. Je savais que ça pesait dans la dose, pas de façon forcément scientifique parce que je n'avais pas fait tourner des modèles, je ne pouvais pas dire "voilà, ça fait tant». Et je leur ai dit "vous ne prenez jamais en compte ce paramètre »(12). Mais les spécialistes s'opposent à l'intégration de cette pratique dans les calculs. II faudra donc les convaincre que cette prise en compte est justifiée. Voyons comment s'est soldée cette discussion. "Ça a été une bataille assez importante (...). Pour eux, c'était quantité négligeable. (...) Et quand l'étude " modes de vie " faite par le Credoc pour le compte de Cogema est arrivée, on s'est aperçu qu'il y avait encore aujourd'hui $12 \%$ des gens dans la région qui pratiquaient cette chose-là. $12 \%$, c'est énorme! Pour eux, c'était une pratique qui n'avait concerné que quelques individus et autrefois. Et là, ils découvrent par un travail qu'ils ont demandé, que, aujourd'hui encore, $12 \%$

(12) Pour bien mesurer l'enjeu de ce débat, précisons que les algues sont utilisées comme bio-indicateurs parce qu'elles concentrent fortement une bonne partie de radioéléments, les amenant donc à la terre lors de l'épandage. des gens font ça. (...) On m'a dit finalement, ton argumentation ne devait pas être complètement fausse... ", (Entretien, ACRO, 1999).

Le récit de la prise en compte des algues dans le calcul des doses révèle ce que l'on doit mobiliser pour convaincre. Pour qu'une pratique locale soit intégrée par les spécialistes du domaine, son porte-parole doit posséder à la fois un savoir scientifique (les algues comme bio-indicateurs), un savoir pratique local (les algues comme amendement) et bénéficier d'appui (le rapport du Credoc) accréditant la pérennité de l'amendement des sols par les algues ${ }^{(13)}$. La démarche adoptée a introduit de la traçabilité dans les hypothèses qui soustendaient les calculs et dans ce cas précis, les algues sont devenues des entités qui balisent le traçage de la production des données par importation d'un savoir local dans le processus d'élaboration de la connaissance générale. Ce qui favorise la prise en compte de cet élément, c'est à la fois une certaine souplesse des hypothèses et des extrapolations faites dans les modèles. L'affaire des algues a, par ailleurs, mis en visibilité les savoirs tacites contenus dans les énoncés scientifiques et les hypothèses implicites qu'elles contiennent, visant par exemple à considérer l'épandage des algues comme une activité désuète. Cet exemple confirme bien l'idée qu'il existe des «... trajectoires sociales et culturellement inscrites dans les analyses scientifiques

(13) En fonction des concentrations de radioéléments dans les algues, les experts évaluent des facteurs de transferts de l'algue au sol et ensuite du sol à la plante. 
des risques 》 (Wynne, 1997 - p. 151). En focalisant leur intérêt sur les hypothèses qui sous-tendent les modèles, l'intervention des non spécialistes éclaire l'incomplétude inhérente à la science et rappelle la façon dont celle-ci produit des connaissances sur des sujets aussi complexes.

\subsection{S'entendre sur des paramètres de modes de vie}

Suite à la remise, en juillet 1999, de résultats qui concluent à un risque radiologique très faible, le GRNC se voit confier en août 2000, une deuxième mission comportant trois volets ${ }^{(14)}$. Le GRNC identifie les pans d'étude qu'il n'a pas abordés et, par là-même, prend acte ${ }^{(15)}$ de critiques internes soulevées par des associatifs - ainsi celles de la CRII-RAD sur l'impact éventuellement leucémogène des rejets chimiques. La réalisation de l'étude de l'impact des rejets chimiques implique de rassembler les données des différents organismes concernés et d'opérer un ajustement entre les cultures scientifiques de la radiologie et de la chimie. Pour ce faire, le GRNC s'ouvre à des experts institutionnels

(14) Ces trois volets sont l'analyse d'incertitude associée au résultat fourni dans la première étape, la comparaison des méthodes de travail du comité britannique Comare avec celle du GRNC et la réalisation de l'étude de l'impact des rejets chimiques des installations nucléaires.

(15) Cette volonté affichée n'est pas une vaine promesse puisqu'elle nécessitera encore deux ans de travail auquel il faut ajouter un an de préparation par l'Institut de Radioprotection et de Sûreté Nucléaire (IRSN) dans le cas de l'analyse d'incertitude. Au total, l'élaboration des réponses aux questions ministérielles aura nécessité cinq ans de travail. et associatifs du chimique, comme I'INERIS, I'Institut de Veille Sanitaire (InVS), l'IFREMER (Institut Français de Recherche pour l'Exploitation de la Mer), l'association « Robin des Bois ». II lui faut également combler la faiblesse des mesures faites dans l'environnement sur les rejets chimiques ${ }^{(16)}$. Un groupe de travail est constitué pour prendre en charge "l'évaluation du risque sanitaire et environnemental dû aux rejets chimiques » et propose notamment des scénarios d'exposition prenant en compte les habitudes alimentaires de groupes d'individus habitant dans le Nord-Cotentin.

Trois scénarios sont proposés par ce groupe de travail : le premier pour l'individu moyen, le second pour l'agriculteur gros consommateur de produits terrestres et le dernier pour le gros consommateurs de produits marins. La définition des valeurs des paramètres de modes de vie s'appuie principalement sur les données de deux études locales récentes du CREDOC. Pour disposer de données sur des effectifs plus importants que ceux du canton de BeaumontHague et afin d'obtenir une évaluation plus fiable, le groupe de travail sanitaire décide d'inclure l'ensemble de l'arrondissement de Cherbourg. Lors de la présentation des scénarios, les associatifs contestent la définition des

(16) Précisons que ce sont les résultats de l'étude d'impact, eux-mêmes liés aux connaissances du moment qui ont orienté les programmes de surveillance de l'environnement imposés par l'administration; enfin, on peut noter que compte tenu de l'évolution progressive des connaissances en la matière et l'imposition d'une étude sanitaire depuis deux ans, les études d'impact doivent être dorénavant plus précises sur ce sujet. 
rations alimentaires au motif qu'elles ne comprennent pas les produits locaux très consommés, comme le cidre, la rhubarbe, le miel ou les noix. Les quatre produits (champignons, miel, gibier, noix) connus pour concentrer les polluants chimiques et les radionucléides devraient être intégrés dans les produis analysés car ils sont l'objet de consommations locales dans le Nord-Cotentin. Par ailleurs, l'utilisation des données pour l'arrondissement de Cherbourg leur paraît contestable car il s'agit de données «urbaines » reflétant mal les pratiques rurales de la zone concernée, notamment par rapport au pourcentage d'autoconsommation et au temps passé à la plage. Ce sont plus globalement les données retenues sur les modes d'approvisionnement, les habitudes alimentaires et le mode de vie des habitants qui font l'objet de controverses.

Un retour sur les débats ouverts par l'utilisation de l'étude CREDOC à partir de laquelle sont bâtis les scénarios permet de comprendre la procédure mise en œuvre pour trouver un accord.

«En fait, en discutant, on s'est rendu compte que l'échantillon était très petit quand on restait centré autour du site. Finalement, il n'y avait qu'une seule personne qui avait été interviewée et qui avait un nourrisson. Et, petit à petit, on s'est rendu compte qu'il y avait un gros biais dû à ce petit échantillonnage et que, par contre, si on passait à l'association autour du site et Cherbourg, on avait là une proportion qui donnait beaucoup plus de sécurité. " (Entretien, EDF, 2002). Mais, les associatifs ont réagi en disant "mais comment pouvez-vous comparer la ville avec la campagne! », forçant les experts à tout reprendre et à relire ensemble les résultats du CREDOC.

«Nous aussi, à EDF, on fait quelques enquêtes sur les rations alimentaires autour de nos sites et on s'est aperçu que, petit à petit, il y avait une homogénéisation des modes de vie entre campagnes et villes, et notamment des modes d'approvisionnement; que le centre Leclerc, qui est à la sortie de la grande ville, est autant fréquenté par des gens de la ville ou des gens de la campagne. Donc je l'avais dit mais le responsable associatif n'avait pas l'air tout à fait convaincu. Et puis on a regardé en détail et on s'est aperçu que si on comparait le mode de vie du petit coin autour des sites et de la ville, y compris en termes de nombre de jours de vacances, on se rendait compte qu'il n'y avait pas de grandes différences... Et il a fini la réunion en reconnaissant qu'il était très surpris. » (Idem).

Le rapport du CREDOC, qui peut s'appuyer sur du tangible et sur des pratiques effectives, permet ainsi de quitter le domaine de l'a priori et des idées reçues. Comme dans l'exemple des algues, mais cette fois en faveur de l'argumentaire des exploitants. Ce rapport met un terme aux discussions et le groupe s'accorde in fine sur la zone entière pour établir les rations alimentaires modèles et les comportements de vie des habitants.

\section{METTRE EN MESURE LES FAIBLES DOSES}

À l'initiative des «Mères en colère ", une campagne internationale de mesures est organisée pour évaluer l'état actuel des niveaux de radioactivité dans la région du Nord-Cotentin 
en associant étroitement les habitants de La Hague ${ }^{(17)}$. On fait appel au réseau MORAL (Mobile Radiological Laboratories), qui regroupe des scientifiques européens expérimentés, pour établir ce bilan. Sur les 15 pays contactés, 10 ont donné une réponse favorable de participation et chaque équipe comprend en général 6 scientifiques d'institution et d'horizon différents. Le projet a été imaginé par l'une des fondatrices du collectif et un membre du GRNC, lui-même responsable du réseau MORAL. Une campagne préliminaire de mesures avait été effectuée en 1998 par cet expert, constituant ainsi une étude de faisabilité de l'exercice réalisé en 2000. C'est aussi une occasion, pour ces scientifiques, d'établir une intercomparaison internationale ${ }^{(18)}$ avec d'autres équipes de spécialistes. Les laboratoires effectuent des prélèvements sur demande des habitants ou de leur propre initiative.

Cette campagne de mesures donnera lieu à la rédaction de deux rapports. Le premier, intitulé « NordCotentin $2000 »{ }^{(19)}$ et agrémenté de photographies, décrit le vécu convivial de l'opération. Sa page de couverture en donne le ton : "Nord-Cotentin 2000 est né d'une rencontre, sur une plage du Cotentin, là où il fait beau cinquante fois par jour, entre un scienti-

(17) Le comité d'organisation comprend la Commission Spéciale et Permanente d'Information (CSPI), I'IPSN, Le Collectif des Mères en colère, l'Office Fédéral de la Santé Publique Suisse (OFSP) et l'OPRI.

(18) Pour une étude sociologique d'une campagne d'intercomparaison, se reporter à Mallard (1996).

(19) Radioactivité de l'environnement, mesures d'intercomparaison, Cherbourg du 9 au 12 octobre 2000. fique suisse un peu poète et une maman normande parfois en colère. La réussite dans le déroulement de cette aventure humaine et scientifique a reposé en permanence sur la confiance mutuelle et le respect entre tous les acteurs. La caravane insolite a sillonné notre région grâce à un formidable esprit de solidarité, de fidélité et d'amitié ». Le second ${ }^{(20)}$, plus technique, décrit la méthodologie adoptée, recense les prises de mesures et apporte des conclusions sur cette campagne d'intercomparaison. Le caractère particulier et novateur de l'exercice NordCotentin 2000 passe par ce lien direct entre scientifiques et habitants pour répondre aux préoccupations de ces derniers suivant en cela la démarche initiée au sein du GRNC.

L'IPSN a participé à l'exercice créant avec l'ACRO une équipe mixte associative-institutionnelle. " Les Mères en colère ", membres du comité d'organisation, ont contribué à l'établissement du programme scientifique. Quand le rapport technique a été rédigé, elles ont fait état de leurs observations et corrigé le document pédagogique comme n'importe quel membre du groupe d'organisation où siégeaient également les chefs de laboratoire et le secrétaire de la $\mathrm{CSPI}^{(21)}$. En ce sens, on peut dire qu'elles sont entrées dans le cercle de l'expertise comme de véritables contre-experts, passant de la mobilisation sociale à une activité tournée vers l'exploration scientifique. La présence

(20) Rapport Nord-Cotentin 2000, résultats et conclusions (2001).

(21) CSPI : Commission Spéciale et Permanente d'Information près de l'Établissement de La Hague. 
de la COGEMA au sein de cette campagne a fait débat pour les « Mères en colère » qui ne voyaient pas leur présence d'un très bon œil. Mais comme l'objectif de la COGEMA était de se comparer avec les autres équipes sur un radionucléide, le krypton 85 , sa participation a finalement été comprise et acceptée sur une partie limitée de la campagne de mesures.

Dans cette opération, conduite pour la première fois sur un territoire faiblement contaminé et en lien étroit avec la population locale, s'est instaurée une situation de don et de contre-don entre la population hébergeante et les mesureurs.

"C'était un exercice extrêmement difficile pour les mesureurs. Ce qui était nouveau pour tous ces gens là aussi, c'était toute une population qui s'intéressait à ce qu'ils étaient en train de faire. Ils logeaient chez l'habitant, ils ne payaient rien chez l'habitant mais en contrepartie ils faisaient des mesures chez eux, dans leur jardin. C'était la première fois que ça se faisait » (Entretien, IRSN, 2002).

" II y a eu toute une série de résultats qui ont nécessité le retour dans les laboratoires parce qu'il s'agissait de prélèvements. Donc c'était un énorme travail et très pédagogique. Je suis allée dans une école où tous les enfants se faisaient mesurer, se faisaient expliquer les choses. Cela a duré une semaine. II y a eu énormément d'articles sur le sujet parce qu'on disait : "La Hague auscultée ». Vous imaginez quarante personnes avec leurs appareils, dans la presqu'île du NordCotentin, en train de faire des mesures dans tous les coins! » (Idem).
Le rapport technique conclut que « le bilan des examens effectués lors de l'exercice Nord-Cotentin 2000 s'avère plutôt rassurant du point de vue de la radioprotection. II n'en demeure pas moins que la poursuite d'une surveillance vigilante et ciblée sur les points d'impact et les radionucléides clés reste justifiée »(22). Cette conclusion se rapproche des recommandations de mise en place de dispositifs de biovigilance ciblés sur les OGM.

L'ouverture de cette expertise critique a des conséquences notables. Ce sont les modèles qui ont été comparés, des mesures qui ont été homogénéisées, de nouveaux radioéléments qui ont été pistés, et finalement le calcul des doses qui a été ajusté. Les chemins de contamination potentielle de l'environnement se sont élargis, modifiés, déplacés. L'expérience du GRNC montre que les associatifs, pourtant moins équipés que les institutionnels, peuvent participer à la définition experte du risque et forcer ces derniers à expliciter les savoirs tacites contenus dans leur évaluation. Toutefois, sur d'autres points, l'asymétrie entre le savoir des uns et des autres reste importante, ce qui limite les marges de manœuvres des associatifs et leur capacité critique. À la suite de ces échanges, les qualifications du risque se stabilisent par un ajustement entre ce que l'on rend visible et ce que l'on parvient à clore, que ce soit par des compromis, des modalités de calculs ou des investigations métrologiques.

Les associations locales du NordCotentin s'efforcent de s'impliquer et

(22) Rapport op cité, p. 25. 
de se faire connaitre comme interlocuteur à part entière dans la surveillance de l'usine de retraitement de la Hague. En amenant au grand jour certains radioélements potentiellement présents dans les rejets normaux ou dus à des incidents de fonctionnement de l'usine, experts et associatifs ont permis une réinterrogation sur les écarts constatés et les remèdes à y apporter et vérifié en retour le caractère acceptable des installations pour l'humain exposé au nucléaire. Ils participent à une amélioration partagée de la compréhension des faibles doses de rayonnements ionisants et du contrôle de la contamination due à des installations les concernant ${ }^{(23)}$. L'action de l'ACRO et des «mères en colère » s'inscrit davantage dans un processus de concernement ${ }^{(24)}$ (Barthe et al., 2002) que dans la revendication militante incarnée par Greenpeace. Cependant, les questions que posent les faibles doses sont si complexes, qu'il y a toujours un moment où les scientifiques, de plus en plus spécialisés, se retrouvent entre eux sur le mode de la science confinée excluant par la même les «mesureurs " de la radioprotection et les groupes concernés.

(23) Cela dit, ce type d'interactions n'est pas toujours aussi productive. A. Roy (2001) montre très bien sur le cas de la Commission du Génie Biomoléculaire que des questions trop pointues et centrées sur la biologie moléculaire, n'ont laissé aucune place aux questions plus générales d'ordre économique, social ou politique.

(24) C'est-à-dire le processus «par lequel des identités émergent, se lient les unes aux autres, pour s'engager parfois (mais sous quelles conditions?) dans des projets de recherche coopérative qui, en retour, par les résultats obtenus, transforment ces identités ». (Barthe et al., p. 64).

\section{CONCLUSION}

Les logiques des acteurs engagés dans l'évaluation experte des risques liés aux faibles doses de rayonnements ionisants visent à faire reconnaître plusieurs modalités de traitement de l'incertitude. En les faisant entrer dans un processus de représentation scientifique, le GRNC contribue à modifier les frontières et les limites de ce qui avait été préalablement défini. L'analyse critique médiée par les associations devient un moteur d'expansion de l'action (Callon et al., 2001; Beck, 2001). À l'intérieur du GRNC, certains estiment alors que l'on a suffisamment réduit l'incertitude pour être en mesure d'agir tandis que d'autres estiment qu'il faut approfondir les investigations pour être en mesure de le faire.

Les conclusions du GRNC soulignent une divergence sur les marges d'incertitude. « Au total, pour les rejets de l'installation de COGEMA, 39 radionucléides sur 75 considérés (52\%) ont été ajoutés à la liste des radionucléides qui ont été fournis par COGEMA au groupe de travail. " (GRNC, rapport de synthèse, 1999). Même si ces ajouts n'entraînent pas d'augmentation notable de l'activité totale rejetée, la composition des rejets a été précisée. La confrontation organisée à l'intérieur de ce comité entre experts et associatifs traduit bien la problématique du risque, laquelle est liée à la capacité de rendre ou non visibles certains phénomènes.

La mission 1 du GRNC consistait à estimer le risque de leucémie radioinduite par reconstitution des doses à partir des différentes sources de rayonnement radio-actif pour la période de 1978 à 1996 et pour la population de 
6656 personnes âgées de 0 à 24 ans du canton de Beaumont La Hague.

Les résultats établissent de façon claire ce que l'on peut imputer aux différentes sources d'exposition:

- sources naturelles : 0,62

- sources médicales : 0,20

- autres : 0,10

- installations nucléaires : 0,0014,

soit un total (arrondi) de 0,83 cas, dont seulement 0,0014 cas serait lié aux installations nucléaires locales.

Concernant le volet réglementaire prospectif de la mission 2 (évaluation des doses aux individus les plus exposé), les deux scénarios de COGEMA et du groupe radioécologie sont présentés; ils montrent que l'on est largement en dessous des limites admises pour le public, malgré des différences notables entre les différents scénarios.

Enfin, la discussion de ces résultats met aussi en évidence une tension entre deux représentations de la précaution (Godard et al., 2002). Une ligne de partage explicite apparaît entre ceux qui estiment pouvoir conclure et d'autres qui, en raison de l'incertitude, estiment préférable de suspendre leur avis. Ainsi, au sujet de la mission 1 est-il mentionné : " $\mathrm{Ce}$ pendant, ce résultat est une estimation moyenne et il convient à ce stade de souligner que les marges d'incertitude n'ont pas été quantifiées. Du fait de cette réserve, certains membres du groupe considèrent ne pas pouvoir à ce stade conclure qu'il est peu probable que les rejets des INB contribuent à l'incidence de leucémie observée dans le canton BeaumontHague. » (rapport cité).
Veiller sur les risques revient finalement à confronter les scientifiques aux questions ou aux points de vue différents et critiques des associations afin d'expliquer leurs choix dans l'élaboration des plans d'échantillonnage, des modèles utilisés et des hypothèses de calculs, après les très nombreux contrôles internes de l'exploitant et externes de l'administration, du public lors des procédures d'autorisation et de la CLI lors du rapport annuel: quelle hypothèse faites-vous, où mesurez-vous, pourquoi prendre ce modèle et pas tel autre, êtes-vous certain d'utiliser le meilleur modèle? Si les associatifs ne peuvent pas opposer des chiffres à ceux des spécialistes, ils les forcent à revoir leur hypothèse de travail, à problématiser autrement l'évaluation des risques. Ainsi, en contrepoint des effets des apprentissages effectués par les non spécialistes, les scientifiques sont conduits à se re-poser et à répondre à des questions analysées antérieurement, ou nouvelles du fait de l'évolution des connaissances scientifiques et d'informations locales ou générales. Pas un geste, pas une pensée qui ne demande à être explicitée afin d'être capable de revenir sur les implicites contenus dans l'évaluation scientifique des risques, ce questionnement externe et indépendant étant de nature à favoriser cette vigilance et ces améliorations permanentes. Autrement dit, l'inquiétude et la surveillance s'élargit en intégrant d'autres acteurs porteurs de savoirs ou de regards singuliers. Ainsi, le comité radioécologie a su construire une expertise crédible sur un sujet sensible parce qu'il a opéré sur plusieurs fronts : la mobilisation de dispositifs métrologiques ouverts 
à des compétences distribuées entre les divers acteurs concernés; l'extension de la gamme des radionucléides à surveiller; un certain rapprochement entre l'épidémiologie et la radioprotection pour assurer un meilleur suivi sanitaire des populations; le dialogue et l'information avec les acteurs locaux, et l'intégration de la « mémoire des lieux » dans la démarche d'objectivation du risque.

\section{RÉFÉRENCES BIBLIOGRAPHIQUES}

Barthe Y., Callon M. \& Lascoumes P., 2002. Réponse à Franck Aggeri. Gérer et comprendre, $\mathrm{n}^{\circ}$ 68: 61-65.

Beck U., 2001. La société du risque. Sur la voie d'une autre modernité. Editions Aubier. Paris: 521 pp.

Callon M., Lascoumes P. \& Barthe Y., 2001. Agir dans un monde incertain. Essai sur la démocratie technique. Le Seuil. Paris: 357 pp.

Cambrosio A. Limoges C. \& Hoffman E., 1992. Expertise as a network: a case study of the controversies over the environmental release of genetically engineered organisms. The culture and power of knowledge, Ed by N.Stehr, R.V. Ericson, Walter de Gruyter, BerlinNew York.

Chateauraynaud F. \& Torny D., 1999. Les sombres précurseurs. Editions EHESS. Paris: $476 \mathrm{pp}$.

Dab W., 1997. Crise de santé publique et crise de la santé publique. Revue Française des Affaires sociales, $\mathrm{n}^{\circ} 3$ 4: $193-200$.

Dab W., 1998. L'information sur la santé et la sécurité est-elle condamnée à être anxiogène ? Risques et peurs alimentaires. Sous la direction de $M$. Apfelbaum. Editions Odile Jacob: 149158.
Estades J. \& Rémy E., 2003. L'expertise en pratique. Les risques liés à la vache folle et aux rayonnements ionisants, Coll. Risques collectifs et situations de crise, ss dir. C. Gilbert. L'Harmattan. Paris.

Godard O., Henry C., Lagadec P., MichelKerjan E. (2002), Traité des nouveaux risques, Précaution, crise, assurance, Paris, Editions Gallimard.

Groupe Radioécologie Nord-Cotentin, 1999. Synthèse sous la présidence de A. Sugier, Estimation des niveaux d'exposition aux rayonnements ionisants et des risques de leucémies associés de populations du NordCotentin. Fontenay-aux-Roses, Institut de Protection et de Sûreté Nucléaire.

Mallard A., 1996. L'interprétation collective des résultats d'une expérience : le cas de l'intercomparaison des instruments scientifiques. Sociologie du travail, Ed. Dunod, $n^{\circ}$ 3: 293-310.

Pobel D. \& Viel J.F., 1997. Case-control study of leukaemia among young people near La Hague nuclear reprocessing plant: the environmental hypothesis revisited. British Medical Journal, Vol. 314 (101): 101-106.

Rapport Nord-Cotentin, 2000. À l'initiative du collectif des "mères en colère ». Mesures d'intercomparaison, Cherbourg du 9 au 12 octobre 2000.

Rapport Nord-Cotentin, 2001. Résultats et conclusions. Cherbourg. 40 p.

Roy A., 2001. Les experts face au risque : le cas des plantes transgéniques. PUF.

Viel J.F., 1998. La santé publique atomisée, radioactivité et leucémies : les leçons de La Hague. Ed. La Découverte. Paris : $217 \mathrm{pp}$.

Wynne B., 1997. Controverses, indéterminations et contrôle social de la technologie. Leçons du nucléaire et de quelques autres cas au Royaume Uni. Le principe de précaution dans la conduite des affaires humaines. Sous la direction d'O. Godard. Editions de la maison des Sciences de l'Homme, Paris. INRA: 149-178. 